\title{
Ultrasonography and computed tomography in patients with right lower quadrant pain: Difficult cases of appendicitis
}

\author{
Adrienne van Randen ${ }^{1,2}$ \\ Wytze Laméris ${ }^{1,2}$ \\ Marja A Boermeester ${ }^{2}$ \\ Julien BCM Puylaert ${ }^{3}$ \\ Jaap Stoker' \\ 'Department of Radiology; \\ ${ }^{2}$ Department of Surgery, Academic \\ Medical Center, University \\ of Amsterdam, Amsterdam, \\ The Netherlands; ${ }^{3}$ Department \\ of Radiology, Westeinde Hospital, \\ The Hague, The Netherlands
}

\begin{abstract}
Acute appendicitis is a common cause of acute abdomen and both computed tomography (CT) and ultrasonography (US) are used in the diagnostic work-up of these patients. In general, imaging has high accuracy in diagnosing acute appendicitis. Although the imaging features of appendicitis are well known, in some patients findings are less conclusive. This pictorial essay will give an overview of difficult US and CT cases of patients suspected of acute appendicitis. Keywords: acute appendicitis, ultrasonography, CT
\end{abstract}

\section{Introduction}

Acute abdominal pain, and in particular right lower quadrant (RLQ) pain, is a common patient presentation at the emergency department (ED), usually requiring immediate diagnostic work-up and care. Acute appendicitis is a common cause for RLQ pain, although many other diagnoses should be considered. Ultrasonography (US) and computed tomography (CT) play an important role for a quick and accurate diagnostic work-up. ${ }^{1}$ With this pictorial essay we aim to give insight into difficult US and CT cases of patients suspected of appendicitis.

\section{Acute appendicitis}

Lifetime risk of developing acute appendicitis is $9 \%$ for males and $7 \%$ for females. ${ }^{2}$ Symptoms generally start with nondescriptive visceral pain in the periumbilical region and anorexia followed by nausea and vomiting. When the disease progresses, typical migration of the pain to the RLQ occurs because of more localized peritoneal inflammation. Patients with a clear-cut physical history can be diagnosed clinically and may not require imaging. ${ }^{3}$ However, diagnosing acute appendicitis may be not that simple, as negative appendectomy rates of $20 \%$ to $30 \%$ have been reported for patients who did not undergo additional imaging after clinical assessment. ${ }^{4,5}$ However, additional usage of US and CT can reduce the negative appendectomy rate significantly to $6 \%{ }^{6}$ Therefore, imaging should be used to confirm or reject the diagnosis appendicitis, and in the latter situation propose an alternative diagnosis.

\section{Imaging techniques}

US with graded compression is a widely accepted technique for evaluation of the appendix. ${ }^{7}$ The $\mathrm{CT}$ technique for evaluating an acute abdomen, with a special interest in the RLQ, involves a CT of the complete abdomen after intravenous contrast medium.

High accuracy (98\%) has been reported for focused appendiceal CT with oral and rectal contrast medium, ${ }^{8}$ but the major disadvantage is the possibility of missing an alternative diagnosis outside the volume imaged or an inflamed appendix at an unusual site (Figure 1). In general, appendicitis can be diagnosed without oral, rectal, or 
A

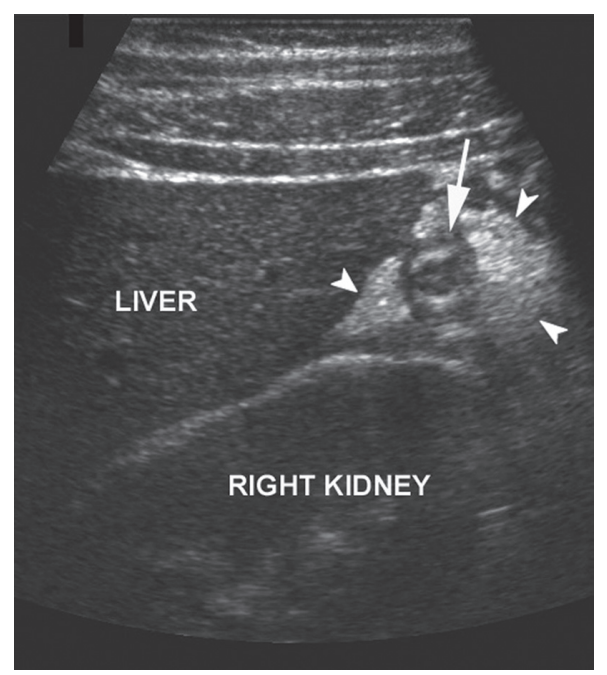

B

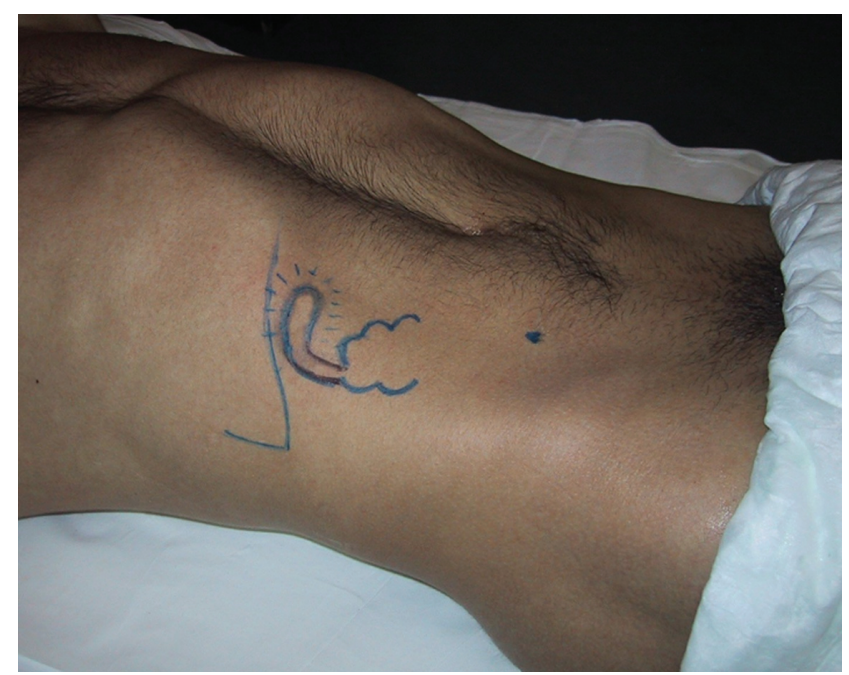

Figure I A) 20-year-old male suspected for acute appendicitis. Ultrasonography demonstrates in this sagittal oblique image an inflamed appendix (arrow) in the right upper quadrant with surrounding fat inflammation (arrowheads) adjacent to the liver and right kidney. B) To guide the operating surgeon, the appendix was marked on the patient's skin. The marked point represents the McBurney point.

intravenous contrast medium. ${ }^{9}$ However, intravenous contrast medium facilitates diagnosing alternative diagnoses and may result in a higher level of confidence, especially when fat interfaces are (almost) absent (slim patients) or obliterated (areas of infiltration). Reconstructed images by multiplanar reformation (MPR) can be of additional help in equivocal CT scans and increases the radiologist level of confidence (Figure 2). ${ }^{10}$ Plain X-ray does not play a role in the work-up of patients suspected with appendicitis. ${ }^{11}$

In current literature CT has higher accuracy compared to US. In a recent meta-analysis of head-to-head comparative studies of US and CT, a summary sensitivity of $91 \%$ was found for CT and of 78\% for US. Specificity was $90 \%$ for CT and $89 \%$ for US. ${ }^{1}$ In another study by Daly and colleagues, 13\% (176/1397) of the CT scans performed for appendicitis were interpreted as equivocal. Of these patients with an equivocal CT $30 \%(n=53)$ had appendicitis. ${ }^{12}$ The sensitivity decreased from $83 \%$ to $64 \%$ if the equivocal scans were interpreted as positive, and the specificity was lowered from $99.8 \%$ to $94 \%$ if the equivocal scans were interpreted as negative.

In our opinion, US should be considered the first modality of choice in female, young, and slender patients, not only because of radiation dose, but also because CT scans of appendicitis in slender patients are more difficult to interpret due to absence of delineating fat. Vice versa, CT is generally better in obese patients because of the delineating fat planes. In very obese patients, the extensive amount of fat causes noise on $\mathrm{CT}$, and will therefore not simplify interpretation of CT images (Figure 3).

\section{Imaging findings of acute appendicitis}

At US the noninflamed appendix appears as a compressible, tubular blind-ending structure with a maximum diameter of $6 \mathrm{~mm}$, without adjacent fat inflammation (Figure 4). In appendicitis the appendiceal diameter is increased, the appendiceal wall is thickened and the surrounding fat is

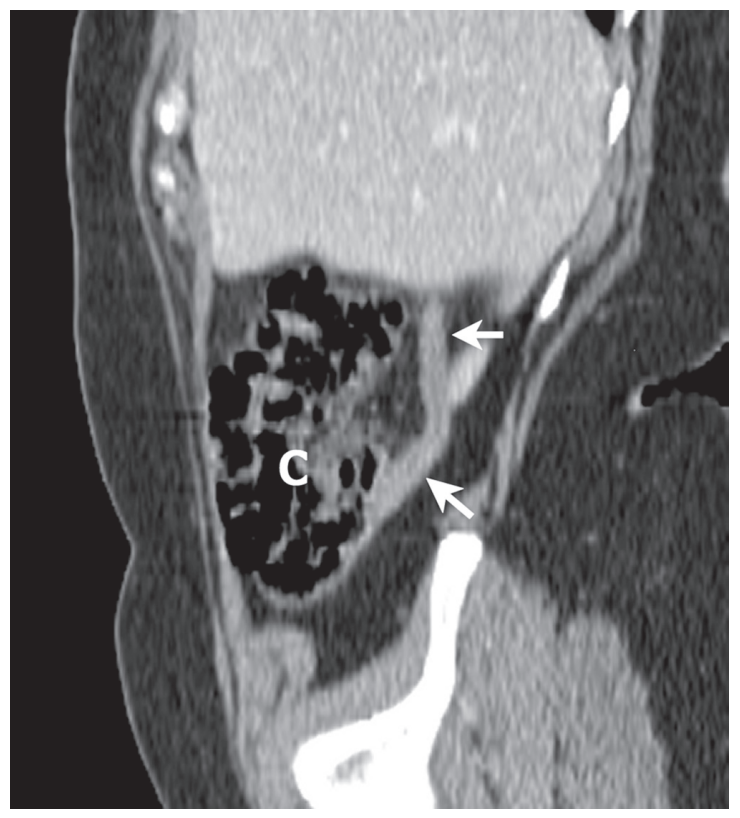

Figure 2 A 39-year-old female with a classical clinical presentation of appendicitis. The appendix could not be visualized on ultrasonography, because of disturbing (bowel) air (not shown). sagittal reformatted computed tomography image after iv contrast medium clearly shows a retrocecal inflamed appendix (arrow); C: cecum. Appendicitis was confirmed at surgery and histopathology. 


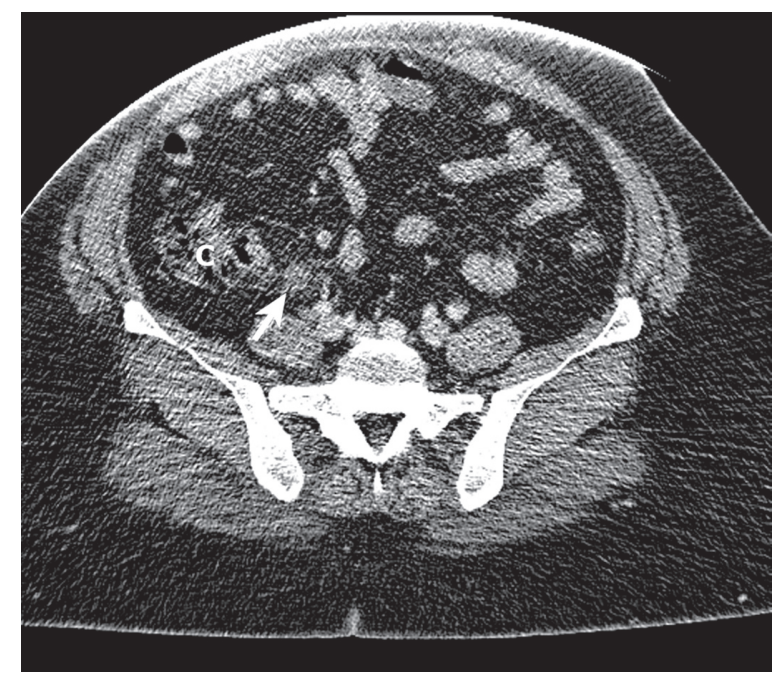

Figure 3 A 37-year-old obese female with a body mass index of 42.7 with histological proven appendicitis. CT after iv contrast medium showed a thickened appendix (arrow) and mild adjacent fat infiltration. C: Cecum.

hyperechoic, and both the appendix and surrounding fat are noncompressible (Figure 5). Appendicitis can only be excluded when the appendix is completely visualized, including the appendiceal tip, and has a normal appearance (Figure 6).

At CT, a noninflamed appendix appears as a tubular blindending structure, usually with a diameter of less than $6 \mathrm{~mm}$, and often containing air. Features of an inflamed appendix at $\mathrm{CT}$ are a tubular, blind-ending structure with a diameter $>6 \mathrm{~mm}$ which is surrounded by inflamed fat (Figure 7). In both US and CT, an appendicolith can be identified in many individuals with and without appendicitis, thus the presence of an appendicolith is not pathognomonic for appendicitis

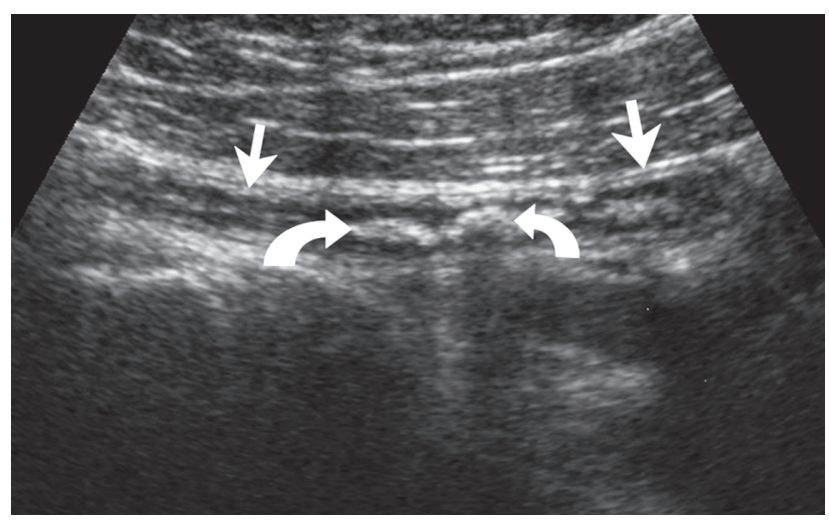

Figure 4 A 23-year-old male with right lower quadrant pain. Ultrasonography (US) shows a nonthickened appendix (arrows) without surrounding infiltration and multiple appendicoliths (curved arrows) and therefore not an US (and final) diagnosis of appendicitis.

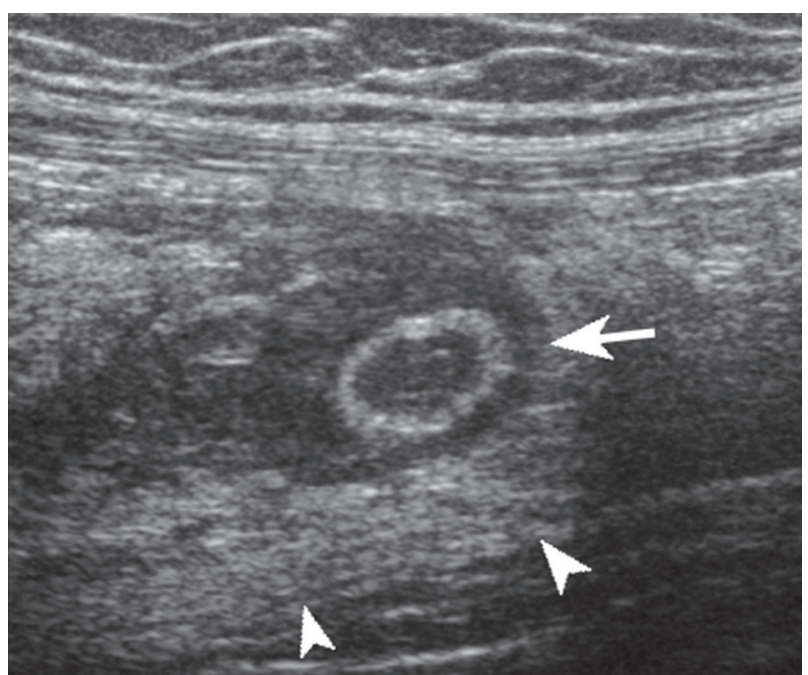

Figure 5 A 49-year-old female suspected of acute appendicitis. Ultrasonography (US) demonstrates a noncompressible, thickened appendix (arrow) surrounded by inflamed mesenteric fat (arrowheads); a clear US diagnosis of acute appendicitis which was confirmed at surgery.

(Figure 4). Also in both, complications can be visible, such as perforation and abscesses.

\section{Difficult cases of US or CT}

US or CT images are difficult to interpret when only one or two of the above mentioned imaging features can be identified. The solitary finding of a thickened appendix, an appendicolith, or fat infiltration adjacent to the appendix are not conclusive signs for the diagnosis acute appendicitis. ${ }^{12}$ These findings may represent normal findings or may reflect early stages of disease or a reactive response to another disease in the RLQ. If the treating physician suspects an early presentation of appendicitis, a 'wait-and-see' policy is

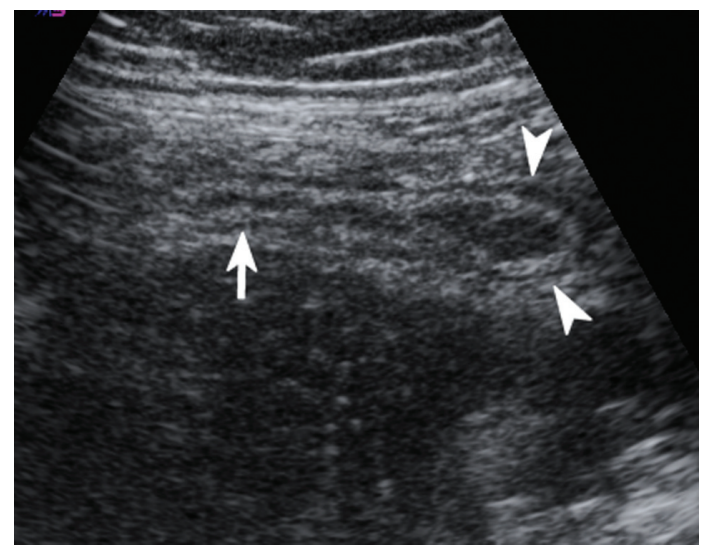

Figure 6 A 43-year-old male with a surgical and histopathological proven inflammation of the appendiceal tip. Ultrasonography images show a normal proximal diameter of the appendix (arrow), but a distal diameter of $8.3 \mathrm{~mm}$ (arrowheads). 


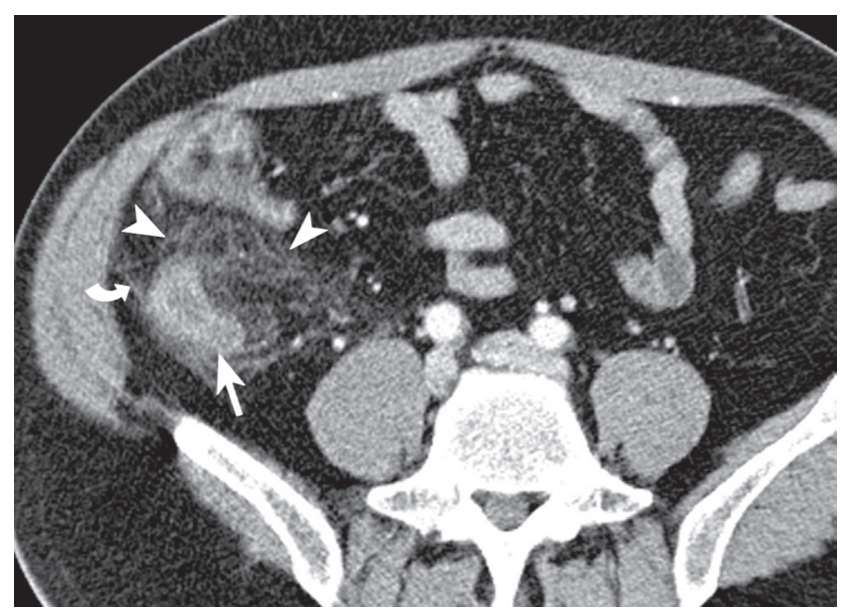

Figure 7 A 49-year-old male suspected for appendicitis. Computed tomography (CT) after iv contrast medium shows a thickened appendix (arrow) with a thickened appendiceal wall, infiltration of the adjacent fat (arrowheads) and a thickened fat plane (curved arrow). Appendicitis was proven at surgery and histopathology.

justified, and the patient maybe asked to return the next day for a second clinical evaluation.

\section{No classical imaging presentation Single imaging finding}

Patients with only a thickened appendix and no clinical or laboratory signs of appendicitis are treated conservatively at our center, because symptoms may resolve spontaneously, and may be clinical irrelevant (Figure 8). Patients with mild fat inflammation adjacent to a nonthickened or nondistended appendix do not have acute appendicitis (Figure 9). In these patients often another diagnosis can be made, while the diagnosis of nonspecific abdominal pain (NSAP) is made when no other clinical and radiological findings are present. Visualization of a normal appendix is crucial for disregarding the diagnosis acute appendicitis.

\section{Multiple imaging findings}

If patients with RLQ pain have multiple, although subtle, inflammatory appendiceal changes or inflammatory changes adjacent to the appendix, the diagnosis appendicitis must be made. If the appendix is thickened and (subtle) fat infiltration is present, acute appendicitis is likely. Appendicitis is also likely when the appendix is somewhat thickened with an enhancing wall and fat infiltration (Figures 10,11). When a thickened appendix is the sole feature, then the diagnosis acute appendicitis is not likely. The only exception is a perforated appendix in which the appendix may not be thickened, but adjacent inflammatory changes are usually extensive in this situation (Figure 12). In patients with mild
A

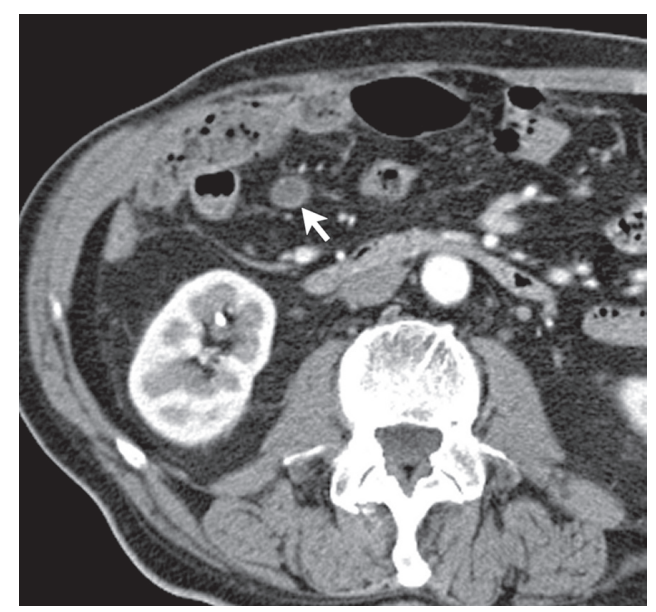

B

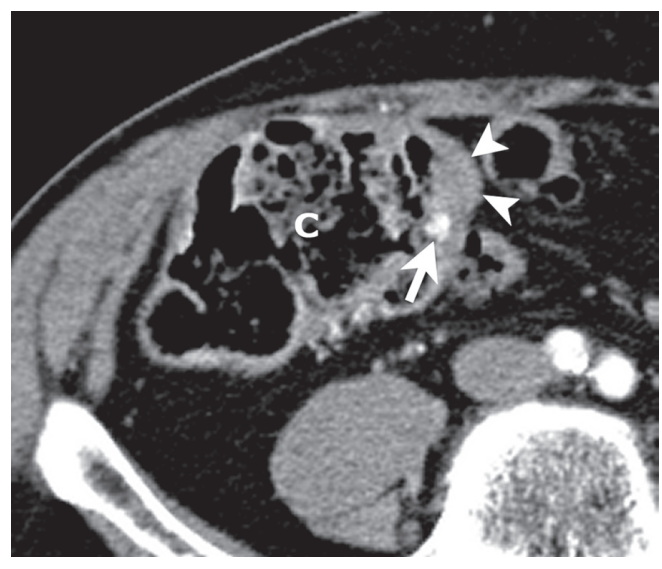

Figure 8 A 75-year-old male suspected for acute appendicitis. A) Computed tomography (CT) after iv contrast showed a fluid filled thickened appendix of $10 \mathrm{~mm}$ diameter (arrow) without inflammation of the adjacent mesenteric fat, more proximal to the cecal orifice the appendiceal lumen was filled with air (not shown) B) An appendicolith was present at the appendiceal orifice (arrow) with cecal wall thickening (arrowheads). On re-examination a few hours later the patient had appetite and pain had decreased. Therefore the patient was discharged home the next day. After more than two years of follow-up, the patient had no recurrent episode of acute right lower quadrant (RLQ) pain. Therefore the final diagnosis of nonspecific abdominal pain (NSAP) was made.

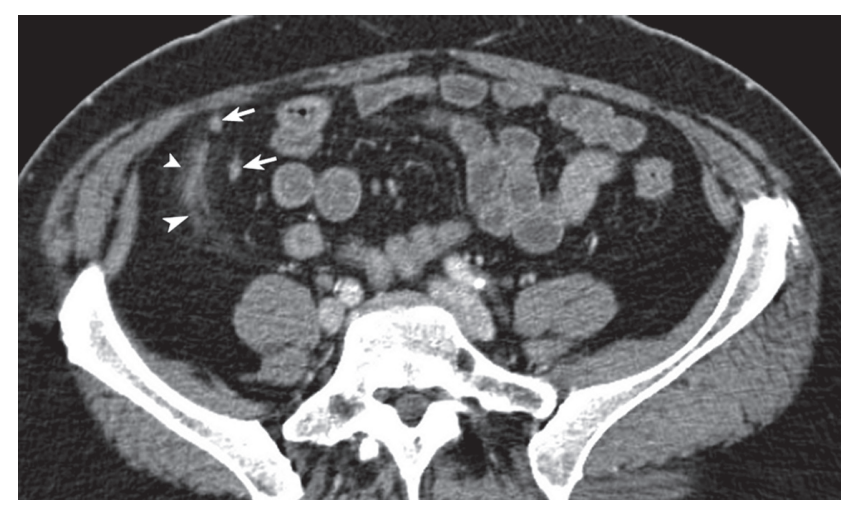

Figure 9 A 60-year-old female with right lower quadrant (RLQ) pain for three days with a final diagnosis of nonspecific abdominal pain after clinical follow-up (up to two years). Computed tomography (CT) after intravenous contrast shows moderate streaky fat infiltration (arrowheads) in the RLQ, but the appendix (arrows) is not enlarged and contains air (not shown). 


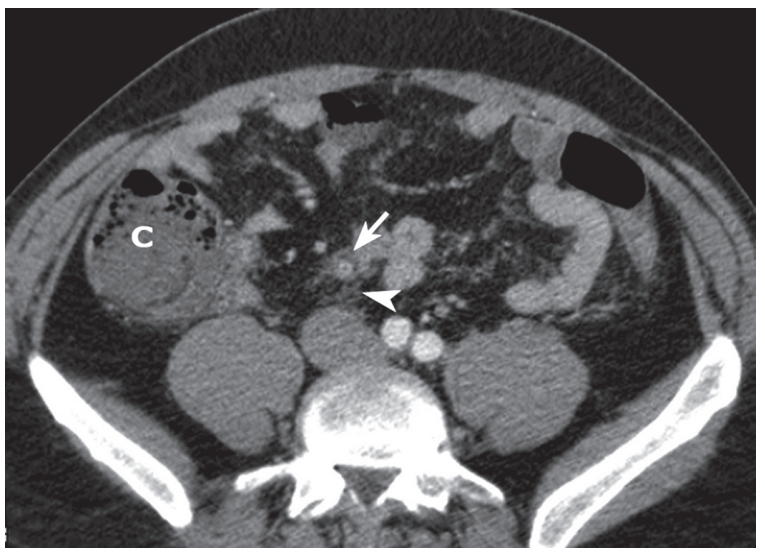

Figure 10 A 38-year-old male with peri-umbilical pain for 16 hours, nausea without vomiting and a low grade temperature was clinically suspected for acute appendicitis. Computed tomography (CT) after intravenous contrast shows a thickened appendix ( $12 \mathrm{~mm}$ ) with an appendicolith (arrow) and subtle fat infiltration (arrowhead). Acute appendicitis was confirmed at surgery and histopathology.

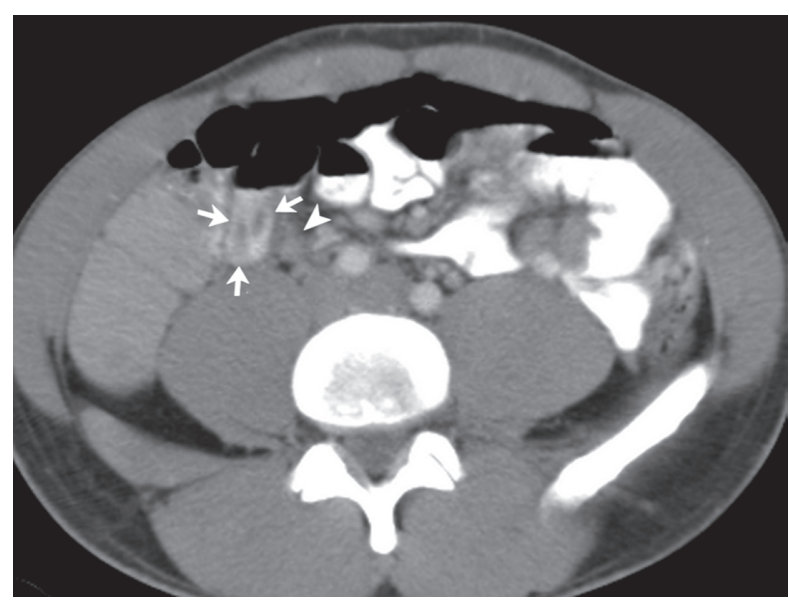

Figure I I A 3 I-year-old male with right lower quadrant (RLQ) pain for two days, nausea without vomiting, and rebound tenderness without guarding, was clinically suspected of acute appendicitis. This computed tomography (CT) image after intravenous (and oral) contrast shows a curved appendix of 9-mm diameter with wall enhancement (arrows) and subtle adjacent fat infiltration (arrowheads). Acute appendicitis was proven by surgery and histopathology.

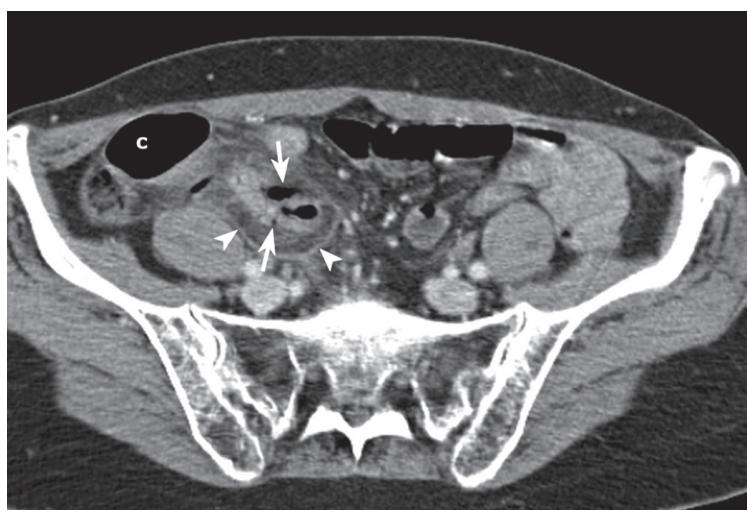

Figure 12 A 55-year-old female clinically suspected for appendicitis shows an perforated appendix (arrow) on this axial computed tomography (CT) image after iv contrast medium. Peri-appendiceal free air is seen (arrows). Also adjacent fat inflammation, with a thickened fat plane is visible (arrowheads). Surgery and histopathology both proved perforated appendicitis in this patient. inflammatory changes in the RLQ and without visualization of the appendix on US and CT, no conclusive diagnosis can be made and management should be based on clinical findings and laboratory findings only and laparoscopy can be considered. However, when the inflammatory changes are extensive (possibly abscesses) the appendix may not be discernable as the fat planes are obliterated (Figure 13). In this situation the diagnosis appendicitis becomes quite likely when no other findings (eg, normal terminal ileum visualized) are present, especially in young men.

Differentiation between terminal ileitis and appendicitis can be difficult when the continuity or blind-ending of an inflamed tubular structure is not visualized. However when a normal-appearing ileum and ileocecal region are visualized, the diagnosis appendicitis becomes very likely (Figure 14).

\section{Secondary appendicitis}

The appendix may become thickened by an adjacent inflammatory condition, such as diverticulitis, a gynecological cause or an inflammation in the ileocecal area (Figure 15). Identification of the primary cause of inflammation is often possible, with the inflammatory response at the primary site being more extensive than adjacent to the appendix.

\section{Atypical location}

A challenge in clinical diagnosing acute appendicitis arises in patients with atypical history and findings at examination because of atypical location of the appendix (Figure 1). In case of a retrocecal appendix, US may have difficulty in diagnosing appendicitis as the appendix is obscured by an

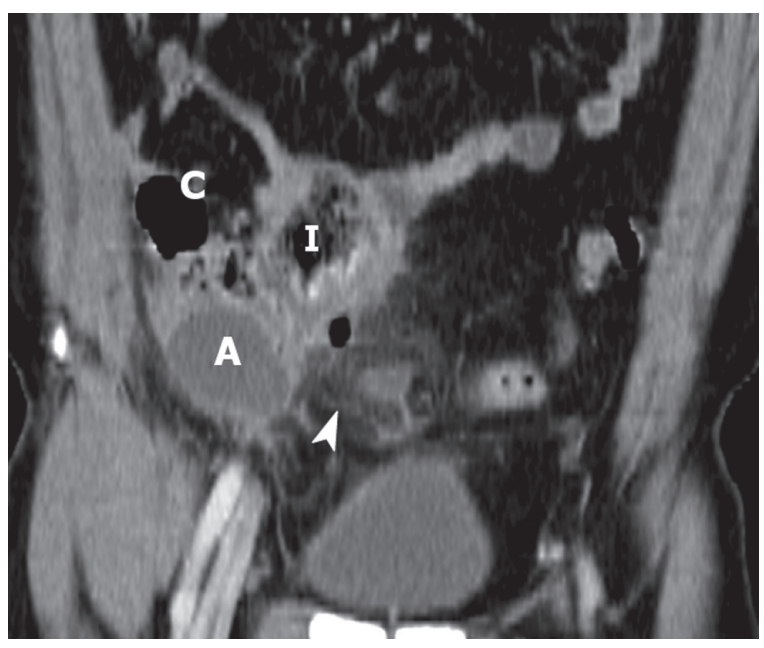

Figure 13 A 44-year-old male with right lower quadrant (RLQ) pain suspected for acute appendicitis. The appendix could not be visualized on computed tomography (CT). This coronal CT image after iv contrast medium demonstrates an abscess (A) with adjacent fat infiltration (arrowhead) located at the suspected site for the appendix and closely related to the cecum (C) and lleum (I) The abscess was drained percutaneously and no appendectomy was performed. 


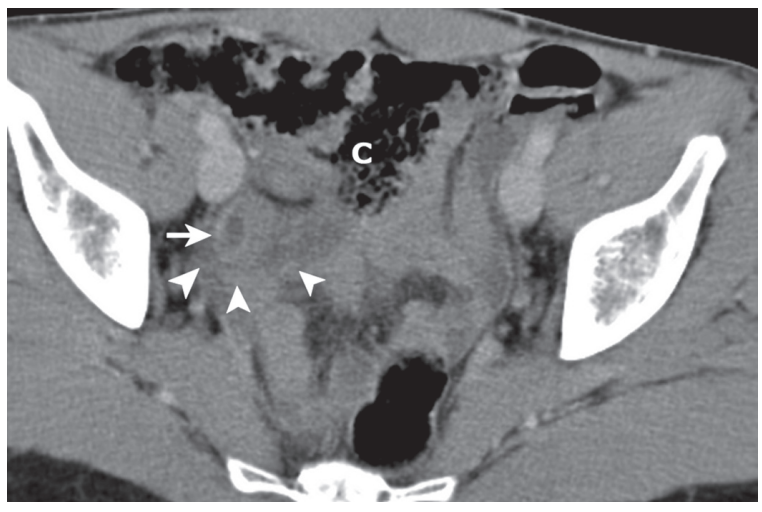

Figure I4 A 33-year-old male with right lower quadrant (RLQ) for one day which migrated from the peri-umbilical region to the RLQ. Computed tomography (CT) after intravenous contrast showed an abnormal tubular structure in the RLQ which could not be visualized completely (arrow). Adjacent to this tubular structure extensive fat infiltration was seen (arrowheads). Furthermore a normal ileum could be identified (not shown). The diagnosis of acute appendicitis was very likely in this male patient, although the blind end of the tubular structure was not visualized. Appendicitis was proven by surgery and histopathology.

air-filled cecum or ascending colon. At CT visualization is not hampered by appendix localization, provided that no focused appendiceal CT of the lower quadrants has been performed, where only part of the abdomen is visualized.

\section{Perforated appendix}

Imaging has limitations in identifying a perforated appendix, although discontinuity of the wall and fluid adjacent to the appendix is suggestive for the diagnosis. ${ }^{13}$ In patients with frank fat infiltration, thickened appendiceal wall, but no distended appendiceal lumen, a perforated appendix should be considered (Figures 12, 15).

\section{Mimics of appendicitis}

Alternative diagnoses mimicking acute appendicitis include right sided diverticulitis (Figure 16), inflammatory bowel disease, epiploic appendagitis, and gynecological causes in women in the reproductive age (Figure 17). Evaluation of specific features of these mimickers are beyond the scope of this pictorial essay, but are well described in the literature. ${ }^{14,15}$

\section{Summary}

The diagnosis of appendicitis can be readily made at US and $\mathrm{CT}$, if multiple imaging features of acute appendicitis are present. In patients with only single or subtle imaging findings such as a thickened appendix without fat infiltration, or merely an appendicolith or fat infiltration adjacent to the appendix, the diagnosis of acute appendicitis becomes unlikely and another cause for their complaints must be sought for. To substantiate these clinical observations, a prospective study should be performed evaluating features of appendicitis at imaging.

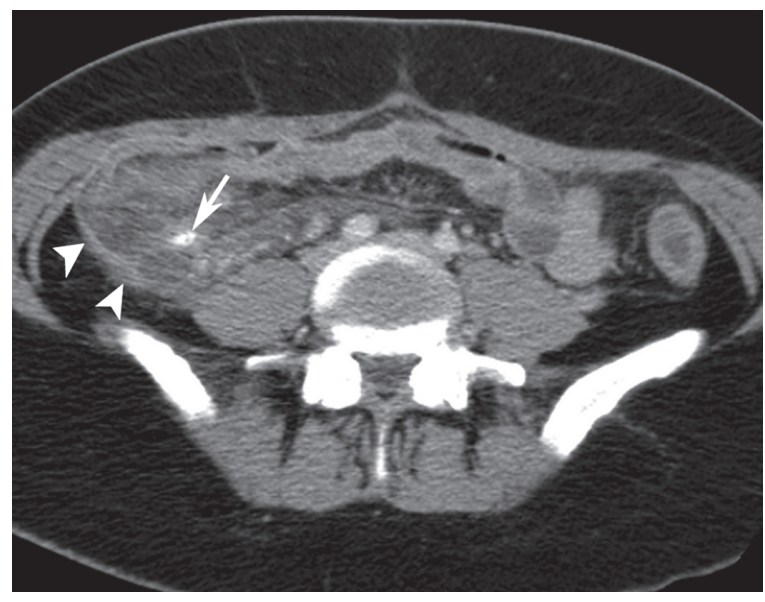

Figure I 5 A 27-year-old female with right lower quadrant (RLQ) pain for three days, clinically suspected for a gynecological disorder or acute appendicitis. Computed tomography (CT) after intravenous contrast showed an appendicolith (arrow), extensive fat infiltration (arrowheads) suggestive of a perforated appendix, although the appendix could not be clearly visualized. Perforated appendicitis was proven at surgery and histopathology.

\section{A}

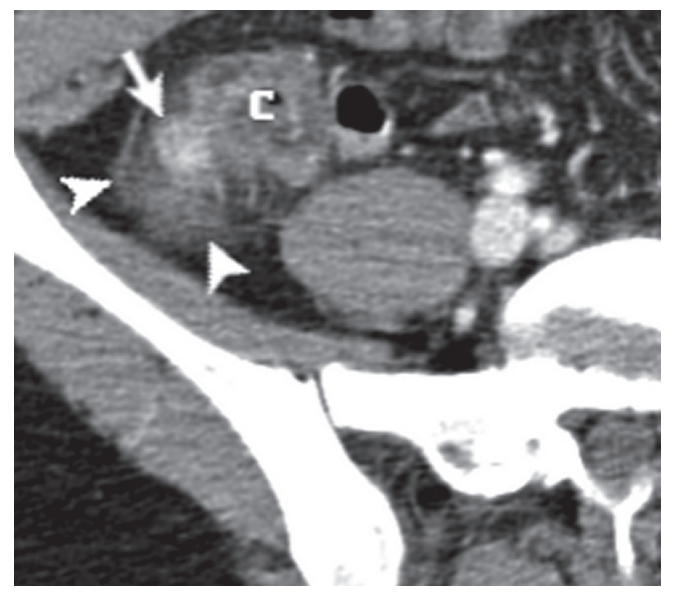

B

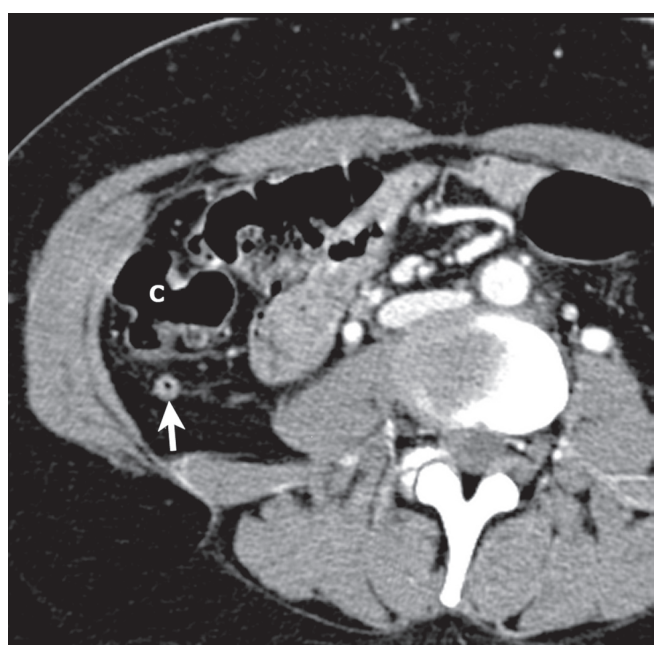

Figure 16 A 25-year-old female with acute right lower quadrant (RLQ) pain. A) A right-sided colon diverticula (arrow) is visible with adjacent fat infiltration (arrowheads), fecolith, wall enhancement on this axial computed tomography (CT) image after i.v. contrast medium; B) Somewhat lower, a nondistended appendix with thickening, containing air, is visible. The appendiceal wall is secondarily inflamed in this patient with right sided diverticulitis. C: Cecum. 


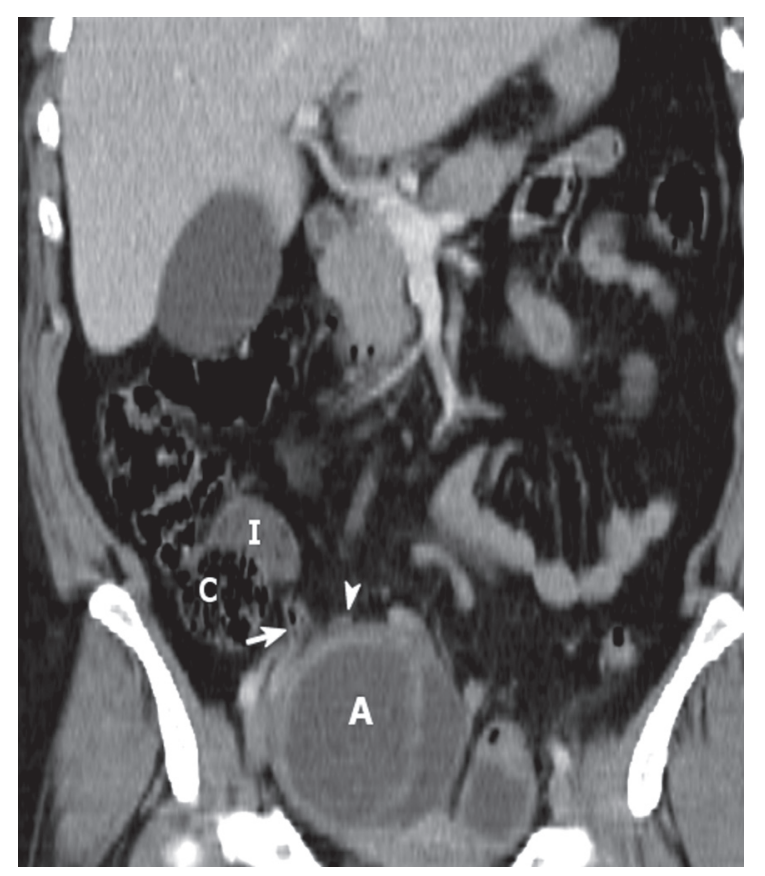

Figure 17 A 42-year-old female with acute abdominal pain suspected for acute appendicitis or diverticulitis. The coronal reformatted image shows a normal appendix (arrow) and a located fluid collection with wall enhancement suggestive for an (ovarian) abscess (A), which was in concordance with the final diagnosis of tubo-ovarian abscess. The mesenteric fat inflammation is visible just cranial of the fluid collection (arrowhead) C: cecum; I: ileum.

\section{Disclosure}

The authors declare no conflicts of interest in this work.

\section{References}

1. van Randen A, Bipat S, Zwinderman AH, Ubbink DT, Stoker J, Boermeester MA. Acute appendicitis: Meta-analysis of diagnostic performance of CT and graded compression US related to prevalence of disease. Radiology. 2008;249(1):97-106.

2. Addiss DG, Shaffer N, Fowler BS, Tauxe RV. The epidemiology of appendicitis and appendectomy in the United States. Am J Epidemiol. 1990;132:910-925.
3. Andersson RE. Meta-analysis of the clinical and laboratory diagnosis of appendicitis. Br J Surg. 2004;91:28-37.

4. Jones PF. Suspected acute appendicitis: trends in management over 30 years. Br J Surg. 2001;88:1570-1577.

5. Raman SS, Osuagwu FC, Kadell B, Cryer H, Sayre J, Lu DS. Effect of $\mathrm{CT}$ on false positive diagnosis of appendicitis and perforation. $N$ Engl J Med. 2008;358:972-973.

6. Florence M, Flum DR, Jurkovich GJ, et al. Negative appendectomy and imaging accuracy in the Washington State Surgical Care and Outcomes Assessment Program. Ann Surg. 2008;248:557-563.

7. Puylaert JB, Rutgers PH, Lalisang RI, et al. A prospective study of ultrasonography in the diagnosis of appendicitis. $N$ Engl J Med. 1987;317:666-669.

8. Rao PM, Rhea JT, Novelline RA, Mostafavi AA, McCabe CJ. Effect of computed tomography of the appendix on treatment of patients and use of hospital resources. N Engl J Med. 1998;338:141-146.

9. Lane MJ, Katz DS, Ross BA, Clautice-Engle TL, Mindelzun RE, Jeffrey RB, Jr. Unenhanced helical CT for suspected acute appendicitis. AJR Am J Roentgenol. 1997;168(2):405-409.

10. Paulson EK, Harris JP, Jaffe TA, Haugan PA, Nelson RC. Acute appendicitis: added diagnostic value of coronal reformations from isotropic voxels at multi-detector row CT. Radiology. 2005;235:879-885.

11. Rao PM, Rhea JT, Rao JA, Conn AK. Plain abdominal radiography in clinically suspected appendicitis: diagnostic yield, resource use, and comparison with CT. Am J Emerg Med. 1999;17:325-328.

12. Daly CP, Cohan RH, Francis IR, Caoili EM, Ellis JH, Nan B. Incidence of acute appendicitis in patients with equivocal CT findings. AJR Am J Roentgenol. 2005;184:1813-1820.

13. Bixby SD, Lucey BC, Soto JA, Theysohn JM, Ozonoff A, Varghese JC. Perforated versus nonperforated acute appendicitis: accuracy of multidetector CT detection. Radiology. 2006;241:780-786.

14. Yu J, Fulcher AS, Turner MA, Halvorsen RA. Helical CT evaluation of acute right lower quadrant pain: part II, uncommon mimics of appendicitis. AJR Am J Roentgenol. 2005;184:1143-1149.

15. Yu J, Fulcher AS, Turner MA, Halvorsen RA. Helical CT evaluation of acute right lower quadrant pain: part I, common mimics of appendicitis. AJR Am J Roentgenol. 2005;184:1136-1142. 
